\title{
Carboxy terminal collagen crosslinks as a prognostic risk factor for fall-related fractures in individuals with established spinal cord injury
}

\author{
Vivien Jørgensen ${ }^{1} \cdot$ Hanne Bjørg Slettahjell ${ }^{1}$ Kirsti Skavberg Roaldsen ${ }^{1,2,3} \cdot$ Emil Kostovski $\mathbb{D}^{1}$
}

Received: 13 February 2019 / Revised: 29 May 2019 / Accepted: 15 June 2019 / Published online: 15 July 2019

(c) The Author(s) 2019. This article is published with open access

\begin{abstract}
Study design Prospective cohort study.

Objective To study associations between specific bone turnover markers and fall-related fractures in individuals with spinal cord injury (SCI).

Setting Rehabilitation Hospital.

Methods Carboxy terminal collagen crosslinks (CTX), type-1 procollagen N-terminal (P1NP), albumin-corrected calcium $\left(\mathrm{Ca}^{2+}\right)$, parathyroid hormone (PTH) and vitamin D were examined in a cohort of 106 participants with SCI at least 1 year post injury. The participants were followed for 1 year monitoring fall-related fractures.

Results In total, 29 out of 106 reported having experienced a fall-related fracture post-injury at baseline, and 5 out of 100 had experienced a fall-related bone fracture during the 1 year follow-up. Our main findings were that high levels of serum CTX increased the odds of being in the fracture group, and that 25-hydroxy vitamin D (25 OHD) levels, $\mathrm{Ca}^{2+}, \mathrm{PTH}$ or P1NP were not associated with being in the fracture group.

Conclusions We here present an association between high-CTX plasma levels at baseline and fall-related fractures reported during a 1-year follow-up among individuals with established SCI. We recommend studies with larger SCI populations before further clinical implications can be drawn.
\end{abstract}

\section{Introduction}

Paralysed muscles and diminished vertical load on bones below the neurological level of injury result in extensive bone loss early after a spinal cord injury (SCI). This results in an accelerated condition of osteopenia or osteoporosis and is a frequent cause of bone fractures among individuals with established SCI [1-4]. Loss of bone strength the first months and years after SCI exceeds loss of bone after prolonged bed rest, and in some individuals the loss equals

Hanne Bjørg Slettahjell

hannebjorg.slettahjell@sunnaaas.no

1 Department of Research, Sunnaas Rehabilitation Hospital, University of Oslo, Oslo, Norway

2 Division of Physiotherapy, Department of Neurobiology, Care Sciences and Society, Karolinska Institutet, Stockholm, Sweden

3 Faculty of Health Sciences, Department of Physiotherapy, Oslo Metropolitan University, Oslo, Norway that of a lifetime in able-bodied [5, 6]. Hormonal changes, low weight load and reduced muscle activity are proposed mediators, in addition to age, gender, family history and lifestyle factors such as smoking, alcohol consumption and vitamin D status. Prevention strategies to reduce fracture incidence are important to avoid additional restrictions in physical function after SCI.

The modified fragility assessment score (FRAX) has been introduces as a tool to pinpoint individuals with a high risk of acquiring fractures [7]. However, the FRAX score is not specific to the SCI population. Sufficient vitamin D levels in combination with dietary calcium are important physiological factors that maintain bone mineral density $[8,9]$. Sub-optimal levels of vitamin D causes bone mineral loss through secondary hyperpara-thyroidism, and low serum levels of 25-hydroxy vitamin D (25 OHD) have been shown to predict hip fracture in the elderly [10]. Individuals with SCI have an increased risk of vitamin D deficiency due to reduced sun exposure, bed rest and low physical activity [11]. Carboxy terminal collagen (CTX), a crosslink peptide sequence of type I collagen, is a biomarker of bone 
turnover. Its serum levels are proportional to osteoclastic activity at the time the blood sample is drawn $[12,13]$. We have previously reported CTX serum concentration among individuals with a post-acute SCI to be 10 times higher than among able-bodied [2], and studies have reported high CTX values as a predictor of fractures in able-bodied [14-17]. There is no data on whether high CTX levels predict fractures in individuals with SCI, or evidence concerning optimal vitamin D status after an SCI, and their association with fractures after SCI. Such data could be of value in clinical practice to identify those at risk. In this study, the aim was to determine if specific bone turnover markers can be used to predict fall-related fractures.

\section{Methods}

\section{Inclusion, setting and data collection procedures}

This 1 year, prospective study was part of a multi-centre study: the Spinal Cords Injury Prevention of Falls (SCIP Falls) Study, conducted at Sunnaas Rehabilitation Hospital, Norway, and Rehab Station Stockholm/Spinalis, Sweden, in collaboration with Karolinska Institutet, Sweden [18-20]. Participants were consecutively recruited at regular followups between February 2013 and February 2014 in the catchment areas of South-East Norway, and participants in the present study constitutes the total Norwegian cohort of the SCIP falls study. Inclusion criteria were: traumatic SCI; at least 1 year post injury; minimum age of 18 years; mastering the Norwegian language and understanding the aim of the project. Individuals that were included were classified according to the International Standards for Neurological Classification of SCI [21]. Exclusion criteria were: motor complete injuries above C5, injuries below L5 level or injuries classified as American Spinal Injury Association (ASIA) Impairment Scale (AIS) E (normal sensory and motor functions) [21]. The outcomes of interest were fall-related fractures and their relation to bone-specific parameters and vitamin D status by 25 OHD levels. A fall was defined as "an unexpected event in which the participants come to rest on the ground, floor, or lower level" $[18,22]$. Falls were monitored for 1 year by sending a text message via an online short message service (SMS -Track ApS, Esbjerg, Denmark) every other week. If a participant's SMS reply was 'yes', a structured telephone interview was conducted within a week, focusing on number of falls, and why, how, when and where the fall happened, as well as possible injuries. All participants were telephoned for 4,8 and 12 months after baseline to maintain compliance and collect fall data. See previous published data for a detailed description [18, 19]. Antecubital vein blood samples were collected from the participants once, in the morning after overnight fast at the beginning of the follow-up period. Serum samples were aliquoted, quick-frozen and stored at $-20{ }^{\circ} \mathrm{C}$ before further analyses. CTX crosslinks (lower detection limit $0.07 \mu \mathrm{g} / \mathrm{l}, \mathrm{CV} 10 \%$ ) and type-1 procollagen $\mathrm{N}$-terminal (P1NP, lower detection limit $5 \mu \mathrm{g} / \mathrm{l}, \mathrm{CV} 2-4 \%$ ) were assayed by an electrochemoluminescence immunoassay using a Roche E 170 module by Roche Diagnostics (F. Hoffmann-La Roche Ltd, Basel, Switzerland). Parathyroid hormone (PTH, lower detection limit $0.5 \mathrm{pmol} / 1$, CV 6-7\%) was measured with a non-competitive immunoluminometric assay using an Immulite 2000 by Siemens Healthcare Diagnostics (Erlangen, Germany). Bone-specific alkaline phosphatase (b-ALP, lower detection limit $9.5 \mu \mathrm{g} / \mathrm{l}$, CV 10-13\%) was measured after immune extraction with a kit from QUIDEL Corp. (San Diego, CA, USA). We measured 25 OHD (lower detection limit $13 \mathrm{nmol} / \mathrm{l}, \mathrm{CV}$ 13-16\%) with a radioimmunoassay from DiaSorin (Stillwater, MN, USA). Phosphate, CRP and albumin-corrected calcium $\left(\mathrm{Ca}^{2+}\right)$ were measured with routine, immunometric assays using the Vitros 250 Chemistry System by Ortho Clinical Diagnostics (Johnson \& Johnson, Auckland, New Zealand).

\section{Statistical analyses}

The statistical analyses were performed with SPSS version 25 (Chicago, IL). Descriptive statistics included median, ranges, means and standard deviations (SD), together with simple tests including Fishers-exact and $\chi^{2}$ tests to compare categorical variables and $t$-tests to compare continuous variables. The participants were divided in a fracture group, i.e., those who had experienced one or more bone fracture(s) during the 1 year follow-up due to a fall, and a non-fracture group. We performed simple correlation tests and a binary logistic regression analysis with fracture group versus non-fracture group as the dependent variable, to study factors that might be associated with having experienced one or more fractures at baseline and after fall during the 1-year follow-up. We excluded variables that explained a similar relationship. Age, gender, body mass index (BMI) and completeness of injury (categorised as wheelchair user or ambulatory, mode of mobility was dichotomised as at least $75 \%$ wheelchair use or ambulatory $[18,20]$ ) were included in the logistic regression with the use of the Enter method. Up to five more variables, were added with the backward likelihood ratio (LR) method: CTX, vitamin D plasma levels, previous fractures, time after injury (y) and alcohol consumption (yes/no). Alcohol consumption was defined as yes if you drink more than 9 (women) and 14 (men) standardised units of alcohol per week (WHO guidelines). If a $p$-value $\leq 0.1$, the variable were included. Values are reported as odds ratios (ORs) with intercept $(B)$ with standard error and $95 \%$ confidence intervals (CIs). We considered $p$-values $<0.05$ to indicate statistical significance. 
Table 1 Baseline characteristics and neurological classification of the participants according to ISNCSCI $(N=106)$

\begin{tabular}{lc}
\hline Male, gender, $n(\%)$ & $86(81)$ \\
Age, mean (SD) & $49(16)$ \\
BMI, mean (SD) & $25(5)$ \\
Years from injury, median (range) & $11(1-51)$ \\
Number of prospective falls, median (range) & $1(0-11)$ \\
Nevrological level, n (\%) & \\
Cervical & $54(51)$ \\
Thoracal 1-6 & $16(15)$ \\
Thoracal 7-12 & $28(26)$ \\
Lumbar & $8(8)$ \\
Americal Impairment Scale (AIS), n (\%) & \\
AIS A,B or C C4 and above & $9(8)$ \\
AIS A,B or C C5-C8 & $14(13)$ \\
AIS A,B or C Th1-S5 & $41(39)$ \\
AIS D any level & $42(40)$ \\
\hline
\end{tabular}

BMI body mass index, ISNCSCI International Standard Neurological Classification of Spinal Cord Injury

\section{Results}

\section{Characteristics of the study participants}

The total study population demographics $(n=106)$ are described in Table 1. Gender ratio was 1:5 for female:male. The age of the participants at the time of the interview ranged from 19 to 83 years (mean (SD); 49 (16) years), and time since injury was between 1 and 51 years; 15 (12) years). BMI ranged from 14 to $\left.40 \mathrm{~kg} / \mathrm{m}^{2} ; 25(5) \mathrm{kg} / \mathrm{m}^{2}\right)$. Neurological level ranged from to $\mathrm{C} 1$ to $\mathrm{L} 3$. Sixty-two participants were defined as wheelchair users and 44 as ambulatory.

\section{Fractures and biomarkers of bone turnover}

Table 2 shows biomarkers of bone turnover assessed in our cohort. All parameters were within the reference range of the able-bodied population. Twenty-nine participants reported at baseline to have experienced a fall-related fracture post-injury. The distribution of fractures was most frequently around the knee or proximal femur. Five individuals experienced six fractures related to falling during the 1-year follow-up, including one femur-, two tibia/fibula-, one costa-, one wristand two hand-bone-fracture(s), see Table 3. Blood markers were not retrieved from six of the participants; however, no fractures were reported from these. Figure 1 show the fracture distribution across CTX blood levels and age, Fig. 2 shows fracture distribution across CTX blood levels and years after injury. CTX plasma levels correlated with years from injury in the wheelchair dependent participants $(r=-0,35, p=$ $0.006)$, but not the ambulatory $(r=-0.002, p=0.898)$. Table $4 \mathrm{a}$ shows the results of the association between
Table 2 Biological markers of bone turnover $(n=100)$

\begin{tabular}{lll}
\hline & Men $(n=86)$ & Women $(n=20)$ \\
\hline PTH $(\mathrm{pmol} / \mathrm{l})$ & $4.4(1.9)$ & $4.1(1.9)$ \\
$\mathrm{Ca} 2+(\mathrm{mmol} / \mathrm{l})$ & $1.2(0.04)$ & $1.2(0.04)$ \\
b-ALP $(\mu \mathrm{g} / \mathrm{l})$ & $32(15)$ & $31(10)$ \\
CTX_I $(\mu \mathrm{g} / \mathrm{l})$ & $0.5(0.3)$ & $0.4(0.1)$ \\
PINP $(\mu \mathrm{g} / \mathrm{l})$ & $55(33)$ & $54(19)$ \\
Vit_D3 $(\mathrm{nmol} / \mathrm{l})$ & $47(20)$ & $53(20)$ \\
\hline
\end{tabular}

Numbers are presented as mean $(\mathrm{SD}), p$-value $=0.05$

$C T X$ carboxy terminal collagen, $P 1 N P$ type-1 procollagen $\mathrm{N}$-terminal, $\mathrm{Ca} 2+$ albumin-corrected calcium, $\mathrm{PTH}$ parathyroid hormone and vitamin D3

different variables on fracture group and non-fracture group during the 1-year follow-up. Our model explained from 9 to $26 \%$ of the relationship between the two groups (fracture and non-fracture). The logistic regression analysis revealed that every unit increase in CTX increased the risk of being in the prospective fracture group $(p=0.021)$, however, the CTX confidence interval (CI) was very wide (95\% CI 2.0-4769.6). Table $4 \mathrm{~b}$ shows the results of the association between different variables on fracture group and non-fracture group at baseline. Our model explained from 23 to $24 \%$ of the relationship between the two groups (fracture and non-fracture). The logistic regression analysis revealed that increased time after injury (y) increased the risk of being in the fracture group $(p<0.001)$ at baseline. We found no association between P1NP, $\mathrm{Ca}^{2+}$, PTH and acquired fractures during the 1-year follow-up. A larger proportion of our study population had sub-optimal levels of $25 \mathrm{OHD}(\leq 50 \mathrm{nmol} / \mathrm{l}), 39$ and $62 \%$ for ambulatory and wheelchair users respectively. The mean values were lower with greater variation during the winter months for both groups. During the summer months, mean values of the wheelchair users were still sub-optimal, whereas the ambulatory participants had satisfactory levels of vitamin $\mathrm{D}$ during these months $(\geq 50 \mathrm{nmol} / \mathrm{l})$. We found no correlation between sub-optimal 25 OHD levels (cut-off level calculated for 30,50 and $70 \mathrm{nmol} / \mathrm{l})$ and fall-induced fractures.

\section{Discussion}

We here describe fall-related fractures over a period of 1 year in 106 individuals with SCI and the association to bone biomarkers in serum. In total, 29 out of 106 reported to have had a fall-related fracture at baseline, and 5 out of 106 individuals had experienced a fall-related bone fracture during the 1-year follow-up. Our main findings were that high levels of serum CTX increased the odds of being in the fracture group and that low 25 OHD levels (cut off 30,50 or $75 \mathrm{nmol} / \mathrm{l}$ ) were not associated with being in the fracture group. CTX serum levels as well as all other markers of 


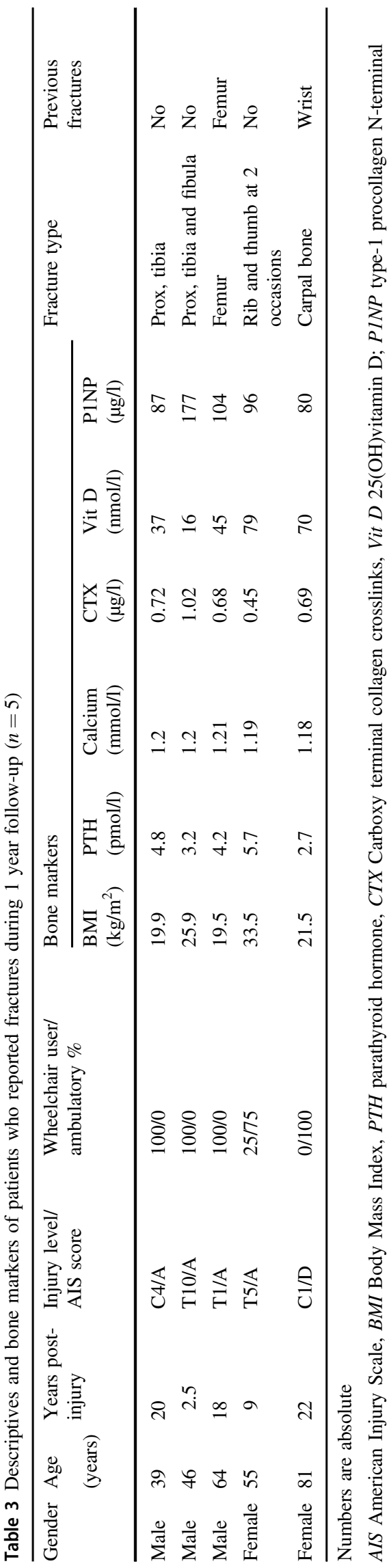

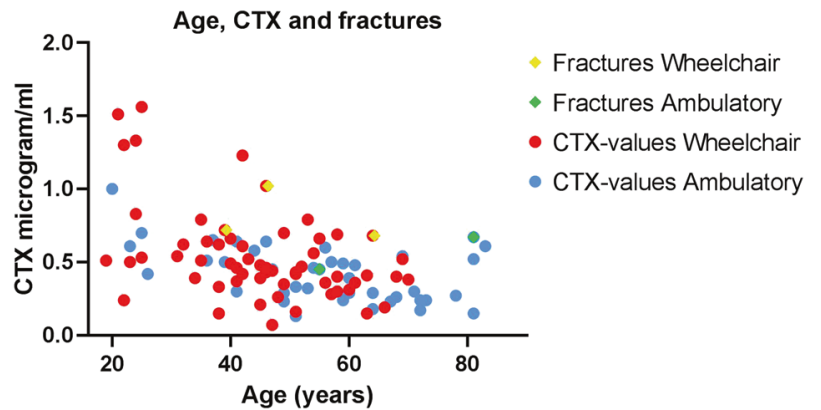

Fig. 1 Fracture distribution across CTX blood levels and age (years). Ambulatory individuals are marked with blue and wheelchair dependent are marked with red dots. Fractures among wheelchair dependent are marked with yellow squares and ambulatory with green squares. WC wheelchair, Amb ambulatory, CTX carboxy terminal collagen

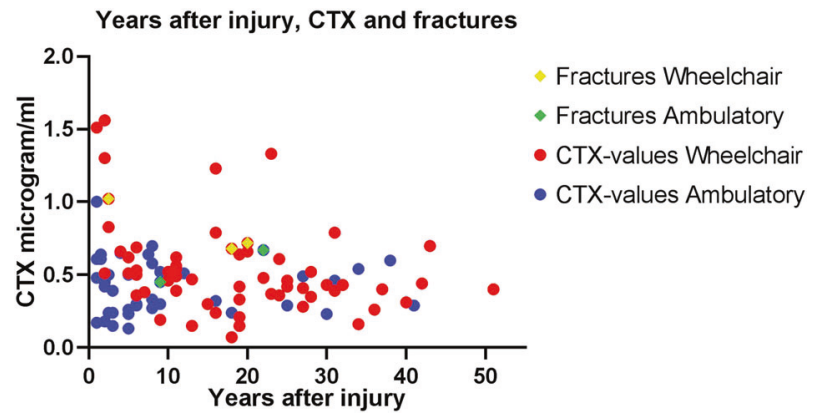

Fig. 2 Fracture distribution across CTX blood levels and time after injury (years). Ambulatory individuals are marked with blue dots and wheelchair dependent are marked with red dots. Fractures among wheelchair dependent are marked with yellow squares and ambulatory with green squares. WC wheelchair, Amb ambulatory, CTX carboxy terminal collagen

Table 4a Prospective fall-related fractures-logistic regression model

\begin{tabular}{|c|c|c|c|c|c|}
\hline \multirow{2}{*}{$\begin{array}{l}\text { Variables associated } \\
\text { with fall-related } \\
\text { fractures }\end{array}$} & \multirow[t]{2}{*}{$B$} & \multirow[t]{2}{*}{ S.E. } & \multirow[t]{2}{*}{$P$-value } & \multirow{2}{*}{$\begin{array}{l}\text { OR } \\
\text { Lower }\end{array}$} & $\begin{array}{l}\text { Confidence } \\
\text { intervals }\end{array}$ \\
\hline & & & & & Upper \\
\hline Age (years) & 0.1 & 0.0 & 0.07 & 1.1 & 1.0 \\
\hline Gender (male/female) & -1.4 & 1.4 & 0.32 & 0.2 & 0.0 \\
\hline BMI (kg/m*m) & 0.0 & 0.1 & 0.89 & 1.0 & 0.8 \\
\hline WC or ambulatory & 1.1 & 1.5 & 0.48 & 3.0 & 61.6 \\
\hline CTX (microgram/l) & 4.6 & 2.0 & 0.02 & 97.9 & $2.0 \quad 4769.6$ \\
\hline
\end{tabular}

Variables included in a multivariate regression model. Mode of mobility was dichotomised as at least $75 \%$ wheelchair use or ambulatory. Model summary: Cox \& Snell $R^{2}$ : 0.089

$B$ intercept, $O R$ odds ratio, $C T X$ carboxy terminal collagen

$* p$-value $<0.05$

bone turnover were within reference range of the ablebodied reference population. According to Marx et al. [12]. CTX plasma levels in the able-bodied population usually range up to $450 \mathrm{pg} / \mathrm{ml}$. In the osteoporosis population, values are commonly $400 \mathrm{pg} / \mathrm{mL}$ to $550 \mathrm{pg} / \mathrm{mL}$ in patients 
Table 4b Fractures after injury-logistic regression model

Variables associated with $B \quad$ S.E. Sig. OR Confidence fall-related fractures

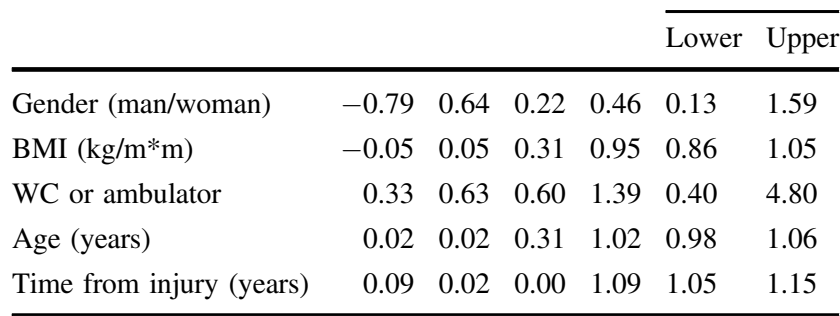

Variables included in a multivariate regression model. Mode of mobility was dichotomised as at least $75 \%$ wheelchair use or ambulatory. Model summary: Cox \& Snell $R^{2}: 0.23$

$B$ intercept, $S E$ standard error, $O R$ odds ratio

$* p$-value $<0.05$

not taking bisphosphonates. All our participants with fractures had $\leq 450 \mathrm{pg} / \mathrm{ml}$. Increased CTX levels increased the risk of being in the fracture group more than variables such as age, ambulation and gender (see Table 4a). However, the CI was wide, most probably due to low total outcome. Finding no associations may merely reflect small numbers of fractures, and thus our results should be interpreted with caution. Even so, based on our study and studies from ablebodied where serum CTX has been found to be associated with fracture risk [12, 16, 23], we believe the association between high-CTX plasma levels and increased fracture risk also to be true for individuals with established SCI. We acknowledge the need for further studies in larger SCI populations. We believe that increased CTX plasma levels should be discussed as a fracture risk factor in addition to other risk factors commonly used in risk assessments scores and also as a monitor of osteoporosis treatment among individuals with SCI. Studies from the able-bodied population have identified CTX in blood to be the reference markers of bone turnover when monitoring osteoporosis treatment [23], and there are today outpatient clinics that monitor osteoporosis treatment with CTX levels also among individuals with SCI. We did not find any correlation between sub-optimal 25 OHD levels and fallinduced fractures. Interestingly, a recent meta-analysis in able-bodied community-dwelling older adults found no association with fracture incidence and supplementation of calcium, vitamin $\mathrm{D}$, or combined calcium and vitamin D [24].

Twenty-seven percentage of our study group reported fractures at baseline with a 5\% fracture rate during the 1year follow-up. Higher numbers of years since injury were associated with a higher risk of fracture post injury at baseline (see Table $4 \mathrm{~b}$ ), which is similar to previous reports $[6,25-30]$. Two out of five individuals in the prospective fall-related fracture group had experienced a previous fracture (see Table 3).This is similar to other studies which show that the patients with SCI who suffer one fracture, are then more likely to experience fracture again [31].

\section{Strengths and limitations}

The strength of this study is the prospective design combined with blood sampling prior to the fracture event. There are relatively few fractures in our study population during the 1-year follow-up, making our statistical calculations prone to misinterpretation. Also, the inclusion of ambulatory subjects could reduce the prognostic value of the dataset for those with complete injuries, and the focus on fall-related fractures may include fractures other than fragility fractures associated with sub-lesional osteoporosis. Furthermore, we did not register fractures not related to falls, such as fatigue and stress fractures. Still, our close follow-up would most likely reveal any non-fall-related fracture. We did analyse the blood samples continuously, and we have no objective measures of osteoporosis of our study participants.

\section{Conclusions}

We here present an association between high-CTX plasma levels at baseline and fall-related fractures reported during a 1-year follow-up among individuals with established SCI. We recommend studies with larger SCI populations before further clinical implications can be drawn.

\section{Data availability}

The datasets generated and/or analysed during the current study are available from the corresponding author on reasonable request.

Acknowledgements We thank all our staff members at the spinal rehabilitation unit at Sunnaas Rehabilitation Hospital for their contributions to implement this study, we would especially like to thank senior consultant Ellen Schaanning for contributing in collecting data. We especially want to thank the study participants.

Funding Sunnaas Rehabilitations Hospital funded the research.

Author contributions VJ was responsible for designing the protocol, writing the protocol and report, screening participants, extracting and analysing data, interpreting results, creating the reference lists, tables and figures and providing feedback on the manuscript. HBS contributed to writing the article, creating tables and figures, interpreting results and providing feedback on the manuscript. KSR was responsible for designing the protocol. She contributed in extracting and analysing the data, interpreting results and providing feedback on the manuscript. EK conducted the statistic analysis, contributed to drafting the manuscript, extracting and analysing the data, creating tables and figures and interpreting results. All the authors have read and approved the final draft of the manuscript. 


\section{Compliance with ethical standards}

Conflict of interest The authors declare that they have no conflict of interest.

Ethical approval We certify that all applicable institutional and governmental regulations concerning the ethical use of human volunteers were followed during the course of this research. This study was approved by the Norwegian Regional Committee for Medical and Health Research Ethics South East (Approval No. 2012/531). The project was carried out in accordance with The Declaration of Helsinki-ethical principles for medical research involving human subjects.

Informed consent All participants provided their written consent prior to inclusion in the study.

Publisher's note: Springer Nature remains neutral with regard to jurisdictional claims in published maps and institutional affiliations.

Open Access This article is licensed under a Creative Commons Attribution 4.0 International License, which permits use, sharing, adaptation, distribution and reproduction in any medium or format, as long as you give appropriate credit to the original author(s) and the source, provide a link to the Creative Commons license, and indicate if changes were made. The images or other third party material in this article are included in the article's Creative Commons license, unless indicated otherwise in a credit line to the material. If material is not included in the article's Creative Commons license and your intended use is not permitted by statutory regulation or exceeds the permitted use, you will need to obtain permission directly from the copyright holder. To view a copy of this license, visit http://creativecommons. org/licenses/by/4.0/.

\section{References}

1. Grimby G, Broberg C, Krotkiewska I, Krotkiewski M. Muscle fiber composition in patients with traumatic cord lesion. Scand J Rehabil Med 1976;8:37-42.

2. Kostovski E, Hjeltnes N, Eriksen EF, Kolset SO, Iversen PO. Differences in bone mineral density, markers of bone turnover and extracellular matrix and daily life muscular activity among patients with recent motor-incomplete versus motor-complete spinal cord injury. Calcified Tissue Int. 2015;96:145-54.

3. Hjeltnes N, Aksnes AK, Birkeland KI, Johansen J, Lannem A, Wallberg-Henriksson H. Improved body composition after $8 \mathrm{wk}$ of electrically stimulated leg cycling in tetraplegic patients. Am J Physiol. 1997;273:R1072-9.

4. Garland DE, Stewart CA, Adkins RH, Hu SS, Rosen C, Liotta FJ, et al. Osteoporosis after spinal cord injury. J Orthop Res 1992;10:371-8

5. Cirnigliaro CM, Myslinski MJ, La Fountaine MF, Kirshblum SC, Forrest GF, Bauman WA. Bone loss at the distal femur and proximal tibia in persons with spinal cord injury: imaging approaches, risk of fracture, and potential treatment options. Osteoporos Int 2017;28:747-65.

6. Edwards WB, Schnitzer TJ. Bone imaging and fracture risk after spinal cord injury. Curr Osteoporos Rep 2015;13:310-7.

7. Cervinka T, Lynch CL, Giangregorio L, Adachi JD, Papaioannou A, Thabane L, et al. Agreement between fragility fracture risk assessment algorithms as applied to adults with chronic spinal cord injury. Spinal Cord 2017;55:985-93.
8. Bouillon R, Van Schoor NM, Gielen E, Boonen S, Mathieu C, Vanderschueren D, et al. Optimal vitamin D status: a critical analysis on the basis of evidence-based medicine. J Clin Endocrinol Metab 2013;98:E1283-304.

9. Dawson-Hughes B, Harris SS, Krall EA, Dallal GE. Effect of calcium and vitamin D supplementation on bone density in men and women 65 years of age or older. New Engl J Med. 1997;337:670-6.

10. Holvik K, Ahmed LA, Forsmo S, Gjesdal CG, Grimnes G, Samuelsen SO, et al. Low serum levels of 25-hydroxyvitamin D predict hip fracture in the elderly: a NOREPOS study. J Clin Endocrinol Metab 2013;98:3341-50.

11. Flueck JL, Perret C. Vitamin D deficiency in individuals with a spinal cord injury: a literature review. Spinal Cord 2017;55:428-34.

12. Marx RE, Cillo JE Jr., Ulloa JJ. Oral bisphosphonate-induced osteonecrosis: risk factors, prediction of risk using serum CTX testing, prevention, and treatment. J Oral Maxillofac Surg 2007;65:2397-410.

13. Rosen HN, Moses AC, Garber J, Iloputaife ID, Ross DS, Lee SL, et al. Serum CTX: a new marker of bone resorption that shows treatment effect more often than other markers because of low coefficient of variability and large changes with bisphosphonate therapy. Calcif Tissue Int. 2000;66:100-3.

14. Dai Z, Wang R, Ang LW, Yuan JM, Koh WP. Bone turnover biomarkers and risk of osteoporotic hip fracture in an Asian population. Bone 2016;83:171-7.

15. Shigdel R, Osima M, Ahmed LA, Joakimsen RM, Eriksen EF, Zebaze R, et al. Bone turnover markers are associated with higher cortical porosity, thinner cortices, and larger size of the proximal femur and non-vertebral fractures. Bone 2015;81:1-6.

16. Johansson H, Oden A, Kanis JA, McCloskey EV, Morris HA, Cooper $\mathrm{C}$, et al. A meta-analysis of reference markers of bone turnover for prediction of fracture. Calcif Tissue Int. 2014;94:560-7.

17. Vasikaran S, Eastell R, Bruyere O, Foldes AJ, Garnero P, Griesmacher A, et al. Markers of bone turnover for the prediction of fracture risk and monitoring of osteoporosis treatment: a need for international reference standards. Osteoporos Int 2011;22:391-420.

18. Jorgensen V, Butler Forslund E, Opheim A, Franzen E, Wahman $\mathrm{K}$, Hultling $\mathrm{C}$, et al. Falls and fear of falling predict future falls and related injuries in ambulatory individuals with spinal cord injury: a longitudinal observational study. J Physiother 2017;63:108-13.

19. Forslund EB, Jorgensen V, Franzen E, Opheim A, Seiger A, Stahle A, et al. High incidence of falls and fall-related injuries in wheelchair users with spinal cord injury: a prospective study of risk indicators. J Rehabil Med 2017;49:144-51.

20. Jorgensen V, Butler Forslund E, Franzen E, Opheim A, Seiger A, Stahle A, et al. Factors associated with recurrent falls in individuals with traumatic spinal cord injury: a multicenter study. Arch Phys Med Rehabil 2016;97:1908-16.

21. Kirshblum SC, Burns SP, Biering-Sorensen F, Donovan W, Graves DE, Jha A, et al. International standards for neurological classification of spinal cord injury (revised 2011). J Spinal Cord Med 2011;34:535-46.

22. Lamb SE, Jorstad-Stein EC, Hauer K, Becker C. Development of a common outcome data set for fall injury prevention trials: the Prevention of Falls Network Europe consensus. J Am Geriatr Soc 2005;53:1618-22.

23. Szulc P, Naylor K, Pickering ME, Hoyle N, Eastell R, Leary E. Use of CTX-I and PINP as bone turnover markers: National Bone Health Alliance recommendations to standardize sample handling and patient preparation to reduce pre-analytical variability. Ann Biol Clin. 2018;76:373-91.

24. Zhao JG, Zeng XT, Wang J, Liu L. Association between calcium or vitamin D supplementation and fracture incidence in community- 
dwelling older adults: a systematic review and meta-analysis. J Am Med Assoc. 2017;318:2466-82.

25. Dionyssiotis Y. Spinal cord injury-related bone impairment and fractures: an update on epidemiology and physiopathological mechanisms. J Musculoskelet Neuron Interact 2011;11:257-65.

26. McKinley WO, Jackson AB, Cardenas DD, DeVivo MJ. Long-term medical complications after traumatic spinal cord injury: a regional model systems analysis. Arch Phys Med Rehabil 1999;80:1402-10.

27. Troy KL, Morse LR. Measurement of bone: diagnosis of SCIinduced osteoporosis and fracture risk prediction. Top Spinal Cord Inj Rehabil 2015;21:267-74.

28. Zehnder Y, Luthi M, Michel D, Knecht H, Perrelet R, Neto I, et al. Long-term changes in bone metabolism, bone mineral density, quantitative ultrasound parameters, and fracture incidence after spinal cord injury: a cross-sectional observational study in 100 paraplegic men. Osteoporos Int 2004;15:180-9.

29. Vestergaard P, Krogh K, Rejnmark L, Mosekilde L. Fracture rates and risk factors for fractures in patients with spinal cord injury. Spinal Cord 1998;36:790-6.

30. Jakimovska VM, Kostovski E, Biering-Sørensen F, Lidal IB. Fractures and musculoskeletal ailments in persons $20+$ years after a traumatic spinal cord injury in Norway. Spinal Cord Ser Cases 2018;4:76.

31. Gifre L, Vidal J, Carrasco J, Portell E, Puig J, Monegal A, et al. Incidence of skeletal fractures after traumatic spinal cord injury: a 10-year follow-up study. Clin Rehabil 2014;28:361-9. 